\title{
Examination of Endoscopic Ultrasonographic Diagnosis for the Depth of Early Gastric Cancer
}

\author{
Kazu Hamada ${ }^{\mathrm{a}, \mathrm{b}}$, Tohru Itoh $^{\mathrm{b}}$, Ken Kawaura ${ }^{\mathrm{b}}$ Hidekazu Kitakata $^{\mathrm{b}}$, Hiroaki Kuno ${ }^{\mathrm{b}}$, Junji Kamai ${ }^{\mathrm{b}}$, \\ Rika Kobayasi ${ }^{b}$, Sadahumi Azukisawa ${ }^{\mathrm{b}}$, Taishi Ishisaka ${ }^{\mathrm{a}}$, Yuta Igarashi ${ }^{\mathrm{a}}$, Kumie Kodera ${ }^{\mathrm{a}}$, \\ Tazuo Okuno a, Takuro Morita ${ }^{a}$, Taroh Himeno ${ }^{a}$, Hiroshi Yano ${ }^{a}$, Toshihiro Higashikawa ${ }^{a}$, \\ Osamu Iritani ${ }^{\text {a }}$, Kunimitsu Iwai ${ }^{\mathrm{a}}$, Shigeto Morimoto ${ }^{\mathrm{a}}$, Masashi Okuro ${ }^{\mathrm{a}, \mathrm{c}}$
}

\begin{abstract}
Background: Endoscopic ultrasonography (EUS) is one of the helpful tools to diagnose depth of early gastric cancer (EGC). In this study, we examined efficiencies of EUS for EGC such as overall accuracy, risk factors of over/under-staging, and accuracies of each invasive distance.

Methods: A total of 403 EGC lesions that could be investigated by EUS during pre-operation and histological diagnosis after endoscopic submucosal dissection (ESD) or surgery were enrolled in this study. For the 403 cases, we analyzed the accuracies of depth by conventional endoscopy (CE) and EUS retrospectively. We evaluated the clinical survey items of CE and EUS which will be described later to compare the differences between "accuracy group" and "over-staging group", and between "accuracy group" and "under-staging group", retrospectively. Additionally, 78 EGC lesions which were confined to the submucosa and for which it was possible to measure accurate invasive distance from the muscularis mucosae were examined for the relationship between preoperative diagnosis of depth by CE and EUS and invasive distance retrospectively.
\end{abstract}

Results: The overall accuracies of both CE and EUS in predicting EGC invasion depth were $87.3 \%$. For CE staging, histological classification was the factor which influenced over-staging. Gastric regions and tumor area were the factors which influenced under-staging of CE. For EUS staging, tumor area was the factor which influenced over-staging, and gastric regions were the factors which influenced under-staging. Both CE and EUS were not sufficient for predicting the lesions confined to $<500 \mu \mathrm{m}$ from the muscularis mucosae because the accuracies of both in predicting depth were less than $50 \%$.

Manuscript submitted February 27, 2021, accepted March 16, 2021

Published online April 27, 2021

aDepartment of Geriatric Medicine, Kanazawa Medical University, Uchinada, Kahoku-gun, Ishikawa 920-0293, Japan

bDepartment of Gastroenterological Endoscopy, Kanazawa Medical University, Uchinada, Kahoku-gun, Ishikawa 920-0293, Japan

${ }^{\mathrm{c} C}$ Corresponding Author: Masashi Okuro, Department of Geriatric Medicine, Kanazawa Medical University, Uchinada, Kahoku-gun, Ishikawa 920-0293, Japan.Email: okuro@kanazawa-med.ac.jp

doi: https://doi.org/10.14740/jocmr4465
However, EUS has a higher accuracy than CE for the lesions confined to $500-2,000 \mu \mathrm{m}$.

Conclusions: The overall accuracies of both CE and EUS in predicting EGC invasion depth were equal, but the contributing factors for over/under-staging were different. Both CE and EUS are not sufficient at present to predict the lesions confined to $<500 \mu \mathrm{m}$ from the muscularis mucosae. However, the accuracy of EUS in predicting them may increase if high-performance EUS systems are developed in the future.

Keywords: Early gastric cancer; Endoscopic ultrasonography; Endoscopy; Depth

\section{Introduction}

Endoscopic resection (ER) such as endoscopic mucosal resection (EMR) and endoscopic submucosal dissection (ESD) are being used widely in the operations for gastrointestinal malignant tumors including early gastric cancer (EGC) [1]. EGC confined to the mucosa (M) is required to be evaluated as the absolute indication with ER, and all EGCs confined to $\geq 500$ $\mu \mathrm{m}$ from the muscularis mucosae (SM2) are not evaluated as absolute indications. On the other hand, some EGCs confined to the submucosa are estimated to be radically cured by ER, such as the lesions which are confined to $<500 \mu \mathrm{m}$ from the muscularis mucosae (SM1), differentiated type, tumor diameter $\leq 3 \mathrm{~cm}$, negative horizontal/vertical margin, and no lymphovascular infiltration [2]. The preoperative diagnosis of EGC invasion depth becomes more important in the future, not only positive/negative submucosal invasion but also accurate invasive distance.

At present, it is standard practice to evaluate preoperative diagnosis of EGC invasion depth in conventional endoscopy (CE) and image-enhanced endoscopy with indigo carmine [3, 4]. However, there are more than a few cases, in particular SM1 cases, in which it is difficult to predict true depth of EGC. Endoscopic ultrasonography (EUS) is one of the helpful tools to diagnose depth of EGC [5, 6], and almost all EGC cases are performed by EUS for preoperative diagnosis of depth in our department. We examined the efficiencies of EUS for EGC in this study, such as overall accuracy, risk factors of over/under- 
staging, and accuracies of each invasive distance.

\section{Materials and Methods}

\section{Patients and methods}

A total of 412 EGC lesions that could be investigated by EUS during pre-operation between August 2008 and April 2017 in Kanazawa Medical University Hospital were enrolled. In nine lesions among the 412, we could not evaluate them appropriately because the condition of them was not good (difficult to evaluate CE or EUS findings by some factors such as aspiration and body motion in five lesions, and difficult to evaluate resected specimens by some factors such as effect of burn and multi-segmentation in nine lesions). Therefore, they were not chosen for this study, and 403 lesions were included in this study. Gastric regions, macroscopic types, histological classification, and depth of tumor invasion were described according to the Japanese Classification [7]. Tumor area was calculated from the endoscopically/surgically resected specimen using the following equation (tumor area $=\pi \mathrm{ab}$, a: long axial radius, b: short axial radius).

\section{Examination 1}

For the 403 cases described above, we analyzed the accuracies of depth by CE and EUS retrospectively, and we evaluated the clinical survey items which influenced over-staging and under-staging of CE and EUS retrospectively. Accuracy was calculated using the following equation (accuracy $=$ the number of cases in which clinical depth of tumor invasion (cT) and each pathological depth of it (pT) agreed/parameter of each pT).

\section{Examination 2}

We categorized the 403 cases described above into three groups; "accuracy group" (cT and pT agreed), "over-staging group" (cT exceeded pT), and "under-staging group" (cT fell pT). We compared between "accuracy group" and "over-staging group", and between "accuracy group" and "under-staging group" to evaluate the clinical survey items which influence over-staging and under-staging of CE and EUS retrospectively. The clinical survey items were gastric regions (upper third (Up)/middle third (Mid)/lower third (Low)), cross-sectional parts of the stomach (lesser curvature(Less)/greater curvature (Gre)/anterior wall (Ant)/posterior wall (Post)), macroscopic types (elevated type (0 - I, 0 - IIa)/flat-depressed type (0 - IIb, 0 - IIc, 0 - III)), tumor area $\left(\mathrm{cm}^{2}\right)$, and histological classification (differentiated type/undifferentiated type).

\section{Examination 3}

There were 78 EGC lesions which confined to the submucosa, and it was possible to measure the accurate invasive distance from the muscularis mucosae for endoscopically/surgically resected specimen. For these, we examined the relationship between preoperative diagnose of depth by CE/EUS and invasive distance retrospectively. Accuracy was calculated using the following equation (accuracy $=$ the number of cases in which cT and each $\mathrm{pT}$ agreed/parameter of each $\mathrm{pT}$ ).

\section{CE staging}

We predicted EGC invasion depth primarily by conventional finding with CE, and secondarily by image-enhanced endoscopy $[3,8]$. Two doctors who were certified endoscopist of Japan Gastroenterological Endoscopy Society for over 5 years discussed and classified them into three stages (M/SM1/SM2) (Figs. 1-3).

\section{EUS staging}

After CE staging, we predicted EGC invasion depth with EUS in the stomach, into which was poured $200-500 \mathrm{~mL}$ of deaerated water through the endoscope. We performed EUS with a miniature sonoprobe system of $20 \mathrm{MHz}$.

We evaluated EGC invasion depth with EUS following Chonan classification [9]. However, in this classification EGCs are classified to two groups (M - SM1 or SM2). We often come across lesions in which have EUS findings between the two groups, and we estimated such lesions as SM1 in this study. Therefore, we classified EGC into three stages (M/SM1/ SM2); EGCs confined to the first to second sonographic layers were categorized as M, EGCs having slight surface irregularity of the third layer were defined as SM1, and EGCs with massive invasion, irregular narrowing, budding or thickening towards both sides into the third layer were guessed as SM2. Just like CE staging, two doctors who were certified endoscopist of Japan Gastroenterological Endoscopy Society for over 5 years discussed and diagnosed those (Figs. 1-3).

\section{Endoscope system}

A high-resolution magnifying endoscope, GIF-H260Z or GIFH290Z (Olympus Medical Systems Corp., Japan) were used for $\mathrm{CE}$ and EUS through the entire period. The processors/ probes which we used for EUS were EU-M2000/UM-3R and EU-ME1/ UM-DP20-25R (Olympus Medical Systems Corp., Japan). The former were used until December 2010, and the latter were used from January 2011.

\section{Histopathology}

The histologic diagnosis was performed by pathologists who were experienced in gastrointestinal cancer. The examination was based on endoscopically resected specimens or surgically resected specimens. As stated above, it was studied as described according to the Japanese Classification [7]. 

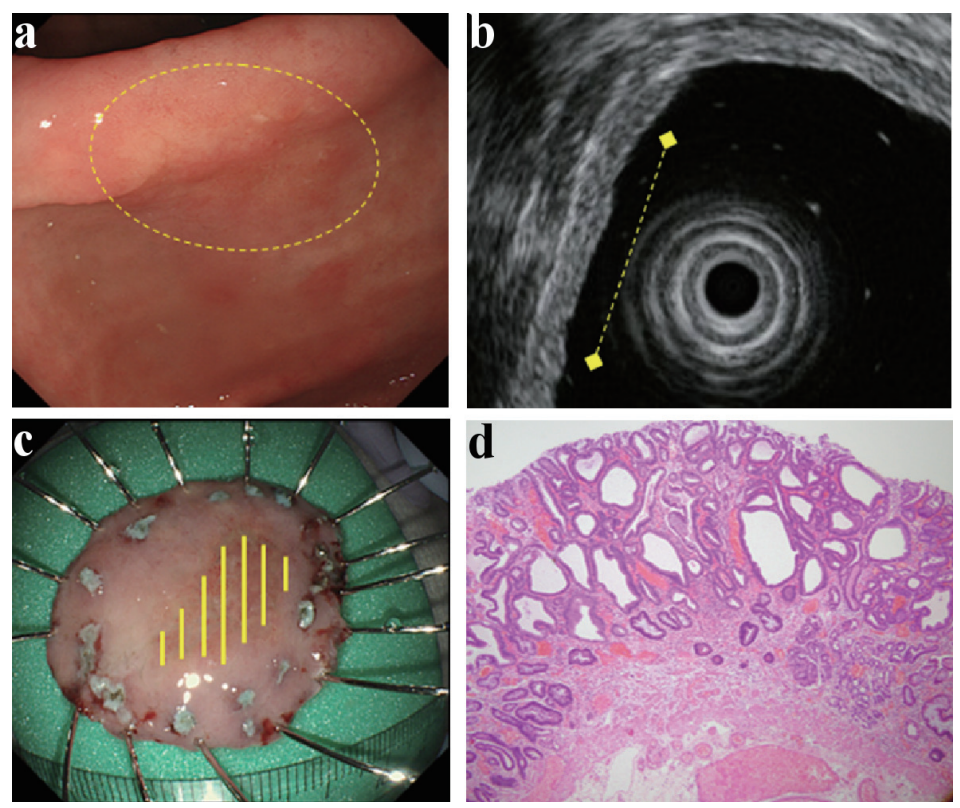

Figure 1. Findings of an EGC confined to the mucosa (M). A depressed type EGC located in the antrum. It was of the differentiated type (tub1 > tub2) and ESD was performed on it. This lesion did not have CE finding from submucosal invasion (a), and it was located in the mucosa with intact submucosa on EUS (b). Therefore, it was predicted to be M by both CE and EUS. This lesion was found to be confined to the mucosa upon histologic examination (c, d). (a) CE (yellow circle: lesion area). (b) EUS (yellow dotted line: lesion area). (c) Resected specimen (yellow line: M). (d) Histological examination of resected specimen (H\&E stain; × 40). EGC: early gastric cancer; ESD: endoscopic submucosal dissection; CE: conventional endoscopy; EUS: endoscopic ultrasonography; M: confined to the mucosa; H\&E: hematoxylin and eosin.
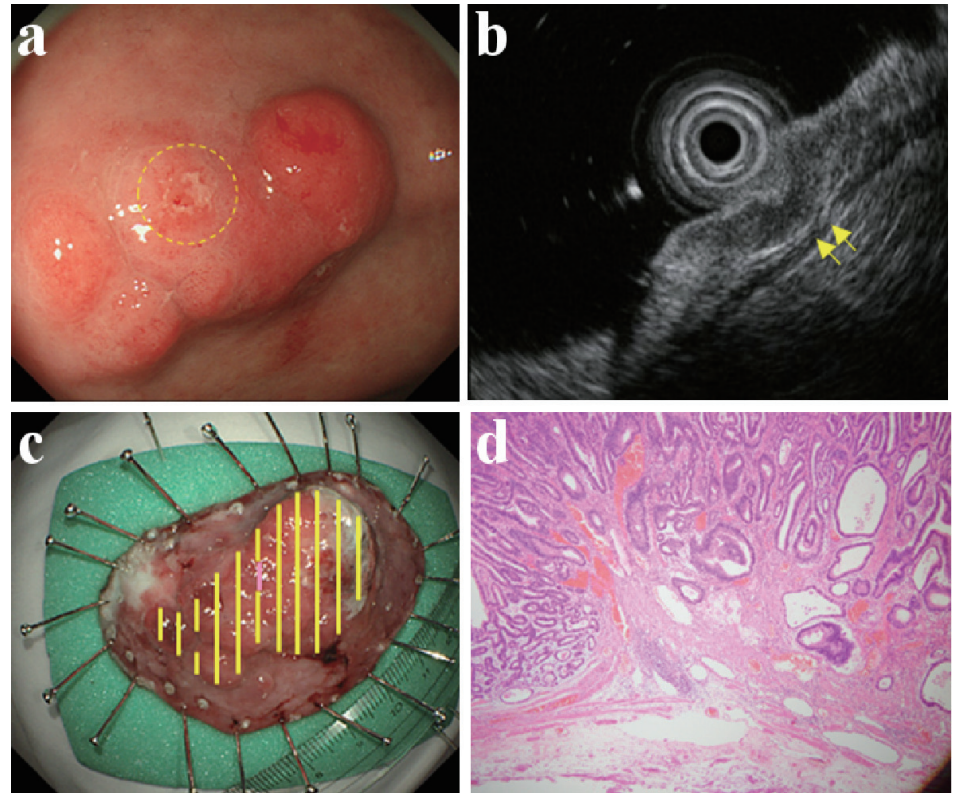

Figure 2. Findings of an EGC confined to $<500 \mu \mathrm{m}$ from the muscularis mucosae (SM1). An elevated type EGC located in the antrum. It was of the differentiated type (tub2 > pap > tub1) and ESD was performed on it. This lesion was found to be a slight/ local erosion by CE (a), and a slight/local crack of the third layer by EUS (b). Therefore, it was predicted to be SM1 by both CE and EUS. This lesion slightly invaded the submucosa at the erosion; found confined to $<500 \mu \mathrm{m}$ from the muscularis mucosae upon histologic examination (c, d). (a) CE (yellow circle: slight/local erosion on the lesion). (b) EUS (yellow arrow: slight/local crack of the third layer). (c) Resected specimen (yellow line: M, violet line: SM). (d) Histological examination of resected specimen (H\&E stain; $\times$ 40). EGC: early gastric cancer; ESD: endoscopic submucosal dissection; CE: conventional endoscopy; EUS: endoscopic ultrasonography; SM: invaded submucosa, SM1: confined to $<500 \mu \mathrm{m}$ from the muscularis mucosae; H\&E: hematoxylin and eosin. 

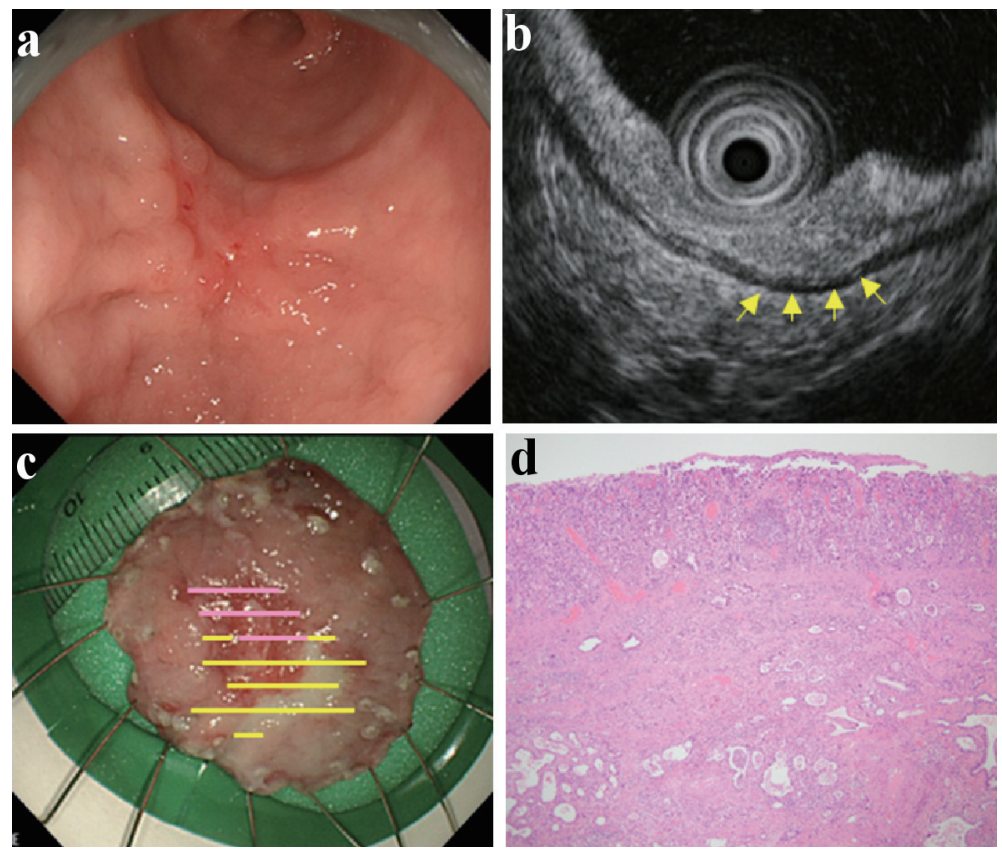

Figure 3. Findings of an EGC confined to $\geq 500 \mu \mathrm{m}$ from the muscularis mucosae (SM2). A depressed type EGC located in the lower gastric body. It was of the differentiated type (tub2) and ESD was performed on it. This lesion was found with fold thickness and fusion of convergent folds by CE (a), and hypoechoic mass with irregular narrowing of the third layer by EUS (b). Therefore, it was predicted to be SM2 by both CE and EUS. There was massive submucosal invasion in the lesion; found confined to $\geq$ $500 \mu \mathrm{m}$ from the muscularis mucosae upon the histologic examination (c, d). (a) CE. (b) EUS (yellow arrow: hypoechoic mass with irregular narrowing of the third layer). (c) Resected specimen (yellow line: M, violet line: SM). (d) Histological examination of resected specimen (H\&E stain; × 40). EGC: early gastric cancer; ESD: endoscopic submucosal dissection; CE: conventional endoscopy; EUS: endoscopic ultrasonography; SM: invaded submucosa; SM2: confined to $\geq 500 \mu \mathrm{m}$ from the muscularis mucosae; H\&E: hematoxylin and eosin.

\section{Statistical analysis}

Statistical analysis was performed using JMP 9 (SAS Institute Japan Ltd.). Comparison of the prevalence was made by univariate analysis using $t$-test or Chi-square test. Furthermore, we performed multiple logistic regression analysis with the factors showing the tendency to involve the $\mathrm{P}$ value $<0.05$ as the confounding factors in our univariate analysis when there were multiple factors in univariate analysis. Statistical significance was taken as $\mathrm{P}$ value $<0.05$.

\section{Ethical considerations}

This study was approved by the Ethics Committee (approval number I-210) at the facility, and was carried out in accordance with the guidelines of Declaration of Helsinki.

\section{Results}

The mean age \pm standard deviation (SD) of the 403 patients was $70.3 \pm 10.0$ years (the range of $30-95$ years), the male/female ratio was $277: 126$, ESD/surgery ratio was $328: 75$, differentiated/undifferentiated ratio was 357:46, and M/SM1/SM2 ratio was 322:18:63 (Table 1).

\section{Examination 1}

The overall accuracies of both CE and EUS in predicting EGC invasion depth were $87.3 \%$ (352/403). The accuracies of CE in each of M, SM1, and SM2 were 94.4\% (304/322), $44.4 \%(8 / 18)$, and $63.5 \%(40 / 63)$. The accuracies of EUS in each of them were $91.6 \%(295 / 322), 44.4 \%$ (8/18), and $77.8 \%$ $(49 / 63)$.

\section{Examination 2}

For CE staging, the lesions of "over-staging group" tended to be larger and more often of the undifferentiated type than "accuracy group", and only histological classification was different from "over-staging group" to "accuracy group". The lesions of "under-staging group" tended to be higher and larger than "accuracy group", and there were significant differences in both gastric regions and tumor area (Table 2).

For EUS staging, "over-staging group" was different from "accuracy group" in the tendency of some items. However, only tumor area was different from "over-staging group" to "accuracy group". Those of "under-staging group" tended to be higher than "accuracy group", and gastric region was the only factor which was significantly different from "under-staging group" in "accuracy group" (Table 3 ). 
Table 1. Clinical Characteristics of Patients and EGC Lesions

\begin{tabular}{ll}
\hline & Patients $(\mathbf{n}=\mathbf{4 0 3})$ \\
\hline Mean age (years) & $71.3(30-95)$ \\
Sex: M/F (\%) & $277 / 126(68.7 / 31.3)$ \\
Treatment: ESD/surgery (\%) & $328 / 75(81.4 / 18.6)$ \\
Gastric regions: Up/Mid/Low (\%) & $49 / 131 / 223(12.2 / 32.5 / 55.3)$ \\
Cross-sectional parts of the stomach: Less/Gre/Ant/Post (\%) & $137 / 73 / 99 / 94(34.0 / 18.1 / 24.6 / 23.3)$ \\
Macroscopic types: elevated type/flat-depressed type (\%) & $162 / 241(40.2 / 59.8)$ \\
Mean tumor area $\left(\mathrm{cm}^{2}\right)$ & $3.486(0.016-98.222)$ \\
Histological classification: differentiated type/undifferentiated type (\%) & $357 / 46(88.6 / 11.4)$ \\
Pathological depth of tumor invasion: M/SM1/SM2 (\%) & $322 / 18 / 63(79.9 / 4.5 / 15.6)$ \\
\hline
\end{tabular}

EGC: early gastric cancer; ESD: endoscopic submucosal dissection; Up: upper third; Mid: middle third; Low: lower third; Less: lesser curvature; Gre: greater curvature; Ant: anterior wall; Post: posterior wall; M: confined to the mucosa; SM1: confined to < $500 \mu \mathrm{m}$ from the muscularis mucosae; SM2: confined to $\geq 500 \mu \mathrm{m}$ from the muscularis mucosae.

\section{Examination 3}

For both CE and EUS, the accuracies in predicting depth were less than $50 \%$ in the lesions confined to $<500 \mu \mathrm{m}$ from the muscularis mucosae, and they were more than $80 \%$ in the lesions confined to $\geq 2,000 \mu \mathrm{m}$. However, there was a difference in the accuracies of both in the lesions confined to $500-2,000$ $\mu \mathrm{m}$. The mean accuracy of CE staging was $39.3 \%$ in these lesions, and it was equal to the accuracy for lesions confined to $<500 \mu \mathrm{m}$. The mean accuracy of EUS staging was $64.3 \%$ in these lesions, and it was between the accuracy for lesions confined to $<500 \mu \mathrm{m}$ and to $\geq 2,000 \mu \mathrm{m}$ (Fig. 4).

\section{Discussion}

The overall accuracies of both CE and EUS in predicting EGC invasion depth were equal, the accuracies for $\mathrm{M}$ were the highest, and the accuracies for SM1 were the lowest in this study. However, EUS tended to be superior to CE in predicting invasion depth of SM2. EUS has a high precision in the diagnosis for T1 - T4 pre-operation staging of the entire gastric cancer [10], but there are some reports of EUS being useful for EGC invasion depth only in limited cases because it does not increase the accuracy compared to CE [11-14]. EUS may diagnose depth of $\mathrm{M} / \mathrm{SM} 1$ lesion exactly, but its accuracy for SM2 lesions is lower than M/SM1 lesions [11, 14, 15]. Additionally, the pre-operation diagnosis of SM1 lesion by EUS is difficult at this stage [16, 17]. The result of this study was almost same content as these reports.

In this study, the risk factor of over-staging was histological classification in $\mathrm{CE}$, and tumor area in EUS. For understaging, they were gastric regions/tumor area in CE, and gastric regions in EUS. The difference was seen in risk factors of over-staging and under-staging in each, and hence it was thought that the knowledge would be useful for improvement of predicting EGC invasion depth by CE and EUS.

As one of the causes of over-staging for EUS, we consider that non-invasion structures such as blood vessel in submucosa are often mistaken as invasion sign. Kikuchi et al reported that there are more percentage of submucosal invasions and frequency of clip use in the EGC group estimated to have reach of vascular structures in submucosa by EUS than non-reach group [18]. They considered that the result occurred because the development and invasion of tumors were followed by the formation of many new blood vessels, and we also think that the hypothesis is broadly correct. On the other hand, they identified submucosal low-echoic spot as a vessel for EUS probe without color Doppler in the examination. In the examination, there is a possibility that they mistook invasion sign as blood vessel in submucosa for some cases, because this EUS finding is a view to be common for both EGC confined to the submucosa and blood vessel in submucosa. Large EGC may have more opportunities for detecting blood vessel in submucosa and mistaking as submucosal invasion by EUS than small EGC. Therefore, over-staging might tend to be caused in large EGC by EUS.

As one of the causes of under-staging for EUS, existence of the lesions which have almost none of the findings to suggest submucosal invasion may participate in it. The cardiac EGCs quite often result in invading submucosa or deeper and causing ulcerative change rapidly though the vertical invasion findings are poor about it for CE [19]. For EUS, there is a study reported that the EGCs in the upper third of the stomach were under-staging more frequently than in other regions [20]. Tsuzuki et al [20] considered that the result occurred because the submucosa tends to be thin and fibrosis in this report. In this study, under-staging tended to be caused in upper lesions by both CE and EUS. This result is the same as in those reports.

For both CE and EUS, the accuracies in predicting depth were less than $50 \%$ in the lesions confined to $<500 \mu \mathrm{m}$ from the muscularis mucosae. Both are not enough to predict SM1 lesion. However, EUS has higher accuracy than CE for predicting invasion depth of slight SM2 confined to $500-2,000$ $\mu \mathrm{m}$. There is a report stated that the EUS limitation of predicting invasive distance for EGC was $1,000 \mu \mathrm{m}$ [16], but we performed EUS mainly with processors/probes which are newer in model than in that report. EUS may improve the accuracy in predicting SM1 lesions more if high-performance EUS systems are developed in the future.

There are several problems in this study. The first is that 


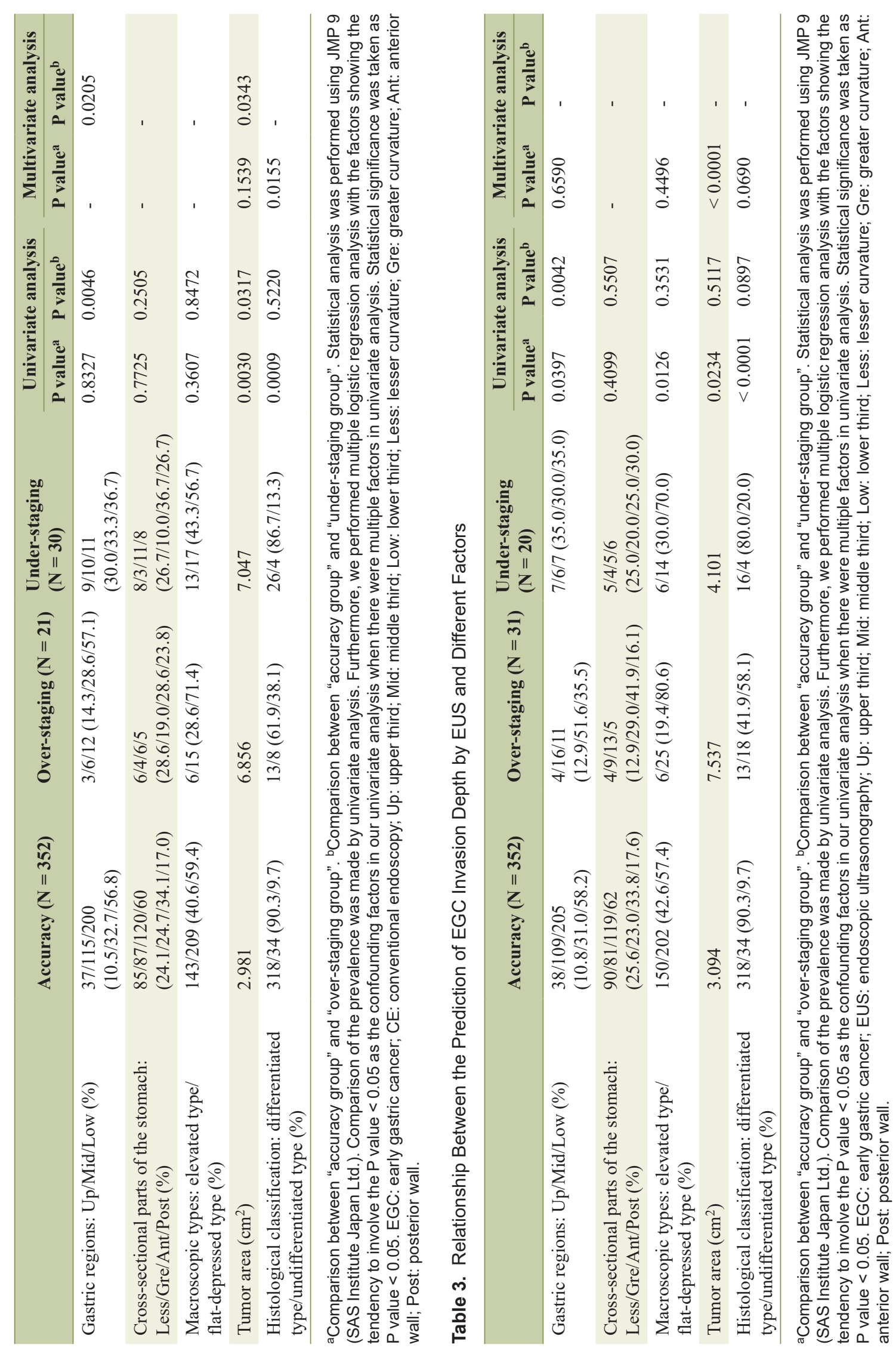



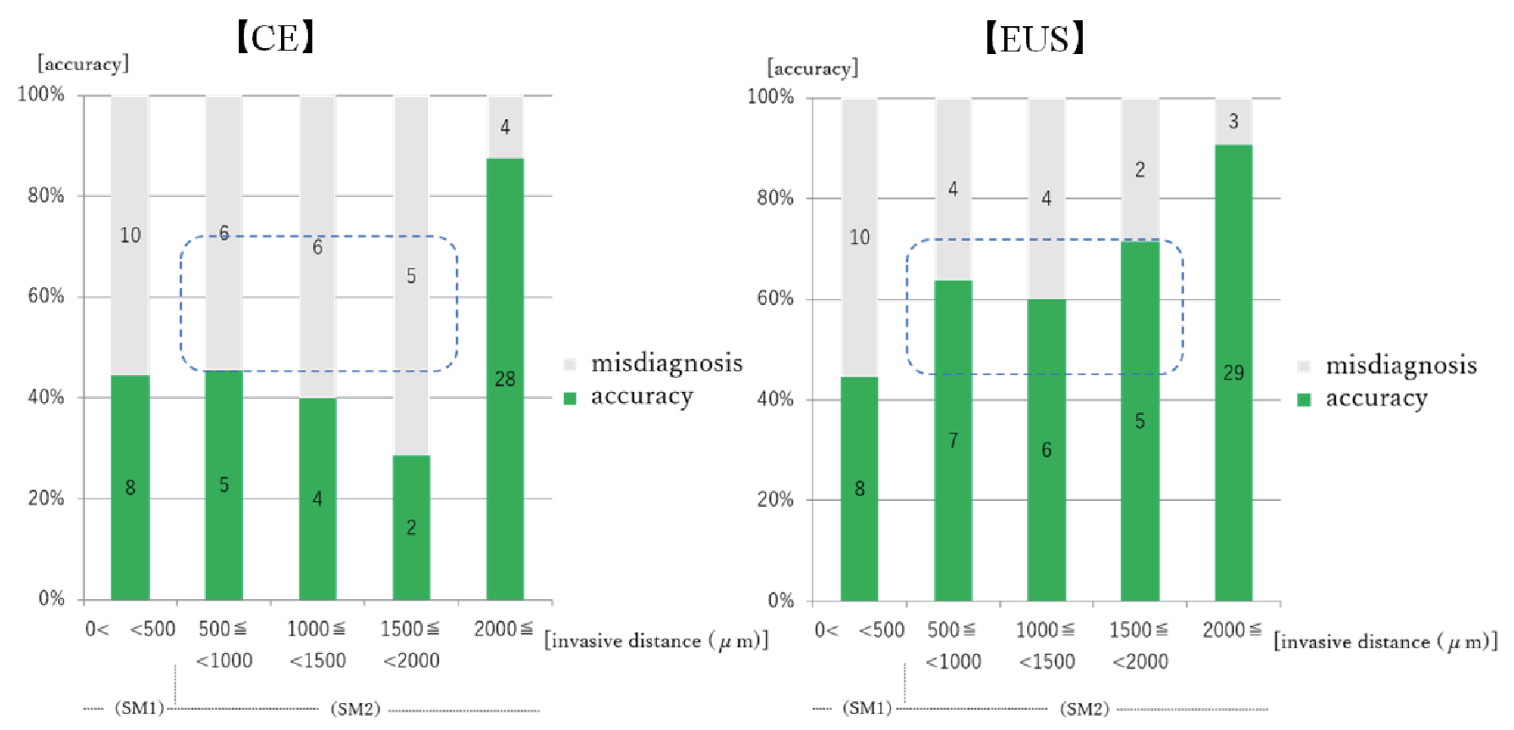

Figure 4. Relationship between prediction of EGC invasion depth by CE/EUS and invasive distance from the muscularis mucosae. EGC: early gastric cancer; CE: conventional endoscopy; EUS: endoscopic ultrasonography. Numeral in the bar graph: number of the cases. For the lesions confined to $500-2,000 \mu \mathrm{m}$, the accuracy of CE staging was about $40 \%$ and it was not superior to the accuracy for the lesions confined to $<500 \mu \mathrm{m}$. The accuracy of EUS staging was $60 \%$ or more in these lesions (blue squares), and was between the accuracy for lesions confined to $<500 \mu \mathrm{m}$ and to $\geq 2,000 \mu \mathrm{m}$.

intratumoral ulcerative findings (UL) were not enrolled as the clinical survey items. Almost all EGC treated in our hospital were consulted from other institutions, they already had some biopsies performed at that time. UL was defined by guideline, but it was often difficult to judge them to be whether UL or biopsy-derived ulcer/scar [2]. Additionally, we sometimes encountered that judgments of UL by CE and histologic diagnosis were extremely different. For those reasons, we decided to remove examination of UL in this study. Secondly, there were a small number of cases confined to $<2,000 \mu \mathrm{m}$ from the muscularis mucosae in "Examination 3" because SM1 - slight SM2 lesions are a minority in the whole EGC. We have to accumulate cases of various EGC and further study this matter.

\section{Conclusions}

The overall accuracies of both CE and EUS in predicting EGC invasion depth were equal, but each contributing factor of over/ under-staging was different. It was thought that the knowledge would be useful for improvement of predicting EGC invasion depth by CE and EUS. Both CE and EUS are not enough to predict SM1 lesions because the accuracies in predicting depth were less than $50 \%$. However, EUS is more useful for predicting invasion depth of slight SM2, confined to $500-2,000 \mu \mathrm{m}$ from the muscularis mucosae than CE. EUS may improve the accuracy in predicting SM1 lesions more if high-performance EUS systems are developed in the future.

\section{Acknowledgments}

We thank all the investigators and staff and participants who participated in this study, for helpful discussions during manuscript development.

\section{Financial Disclosure}

None to declare.

\section{Conflict of Interest}

The authors declare no conflict of interest.

\section{Informed Consent}

Patients were not required to give informed consent to the study because the retrospective analysis used anonymous clinical data that were obtained after each patient agreed to the treatment schedule by written consent.

\section{Author Contributions}

Kazu Hamada wrote the manuscript; Masashi Okuro and Ken Kawaura collected data for review; all authors read and approved the final version of the manuscript.

\section{Data Availability}

The data supporting the findings of this study are available 
from the corresponding author upon reasonable request.

\section{References}

1. Soetikno R, Kaltenbach T, Yeh R, Gotoda T. Endoscopic mucosal resection for early cancers of the upper gastrointestinal tract. J Clin Oncol. 2005;23(20):4490-4498.

2. Japanese Gastric Cancer Association. Japanese gastric cancer treatment guidelines 2018 (5th edition). Gastric Cancer. 2021;24(1):1-21.

3. Choi J, Kim SG, Im JP, Kim JS, Jung HC, Song IS. Endoscopic prediction of tumor invasion depth in early gastric cancer. Gastrointest Endosc. 2011;73(5):917-927.

4. Abe S, Oda I, Shimazu T, Kinjo T, Tada K, Sakamoto T, Kusano C, et al. Depth-predicting score for differentiated early gastric cancer. Gastric Cancer. 2011;14(1):35-40.

5. Choi J, Kim SG, Im JP, Kim JS, Jung HC, Song IS. Is endoscopic ultrasonography indispensable in patients with early gastric cancer prior to endoscopic resection? Surg Endosc. 2010;24(12):3177-3185.

6. Okada K, Fujisaki J, Kasuga A, Omae M, Yoshimoto K, Hirasawa T, Ishiyama A, et al. Endoscopic ultrasonography is valuable for identifying early gastric cancers meeting expanded-indication criteria for endoscopic submucosal dissection. Surg Endosc. 2011;25(3):841-848.

7. Japanese Gastric Cancer Association. Japanese classification of gastric carcinoma: 3rd English edition. Gastric Cancer. 2011;14(2):101-112.

8. Sano T, Okuyama Y, Kobori O, Shimizu T, Morioka Y. Early gastric cancer. Endoscopic diagnosis of depth of invasion. Dig Dis Sci. 1990;35(11):1340-1344.

9. Chonan A, Mishima T, Miyake N, Ishibashi J, Nakahori M, Matsuda T, Takabayashi H, et al. Endoscopic ultrasonographic diagnosis of depth of invasion of early gastric cancer. Stomach Intest. 2009;44(4):623-635 (in Japanese).

10. Puli SR, Batapati Krishna Reddy J, Bechtold ML, Antillon MR, Ibdah JA. How good is endoscopic ultrasound for TNM staging of gastric cancers? A metaanalysis and systematic review. World J Gastroenterol. 2008;14(25):4011-4019.

11. Kim SJ, Choi CW, Kang DH, Kim HW, Park SB, Nam HS, Shin DH. Factors associated with the efficacy of min- iprobe endoscopic ultrasonography after conventional endoscopy for the prediction of invasion depth of early gastric cancer. Scand J Gastroenterol. 2017;52(8):864-869.

12. Choi J, Kim SG, Im JP, Kim JS, Jung HC, Song IS. Comparison of endoscopic ultrasonography and conventional endoscopy for prediction of depth of tumor invasion in early gastric cancer. Endoscopy. 2010;42(9):705-713.

13. Yanai H, Noguchi T, Mizumachi S, Tokiyama H, Nakamura H, Tada M, Okita K. A blind comparison of the effectiveness of endoscopic ultrasonography and endoscopy in staging early gastric cancer. Gut. 1999;44(3):361365.

14. Watari J, Ueyama S, Tomita T, Ikehara H, Hori K, Hara $\mathrm{K}$, Yamasaki $\mathrm{T}$, et al. What types of early gastric cancer are indicated for endoscopic ultrasonography staging of invasion depth? World J Gastrointest Endosc. 2016;8(16):558-567.

15. Tsujii Y, Kato M, Inoue T, Yoshii S, Nagai K, Fujinaga T, Maekawa A, et al. Integrated diagnostic strategy for the invasion depth of early gastric cancer by conventional endoscopy and EUS. Gastrointest Endosc. 2015;82(3):452459.

16. Chonan A, Mishima T, Ando M, Tamura T, Atami M, Mochizuki F. Endoscopic ultrasonographic diagnosis of the depth of invasion of gastric cancer. Stomach Intest. 2001;36(3):341-350 (in Japanese).

17. Isobe S, Yoshino J, Inui K, Wakabayashi T, Okushima K, Kobayashi T, Miyoshi H, et al. Endosonographic diagnosis of the depth of cancerous invasion of differentiated type gastric cancer. Stomach Intest. 2007;42(1):79-87 (in Japanese).

18. Kikuchi D, Iizuka T, Hoteya S, Yamashita S, Nakamura M, Kuroki Y, Mitani T, et al. Usefulness of endoscopic ultrasound for the prediction of intraoperative bleeding of endoscopic submucosal dissection for gastric neoplasms. J Gastroenterol Hepatol. 2011;26(1):68-72.

19. Igari T, Takizawa T, Koike M, Funada N, Hijima T. Growth and spread pattern of cardiac cancers. Stomach Intest. 2001;36(5):651-661 (in Japanese).

20. Tsuzuki T, Okada H, Kawahara Y, Nasu J, Takenaka R, Inoue M, Kawano S, et al. Usefulness and problems of endoscopic ultrasonography in prediction of the depth of tumor invasion in early gastric cancer. Acta Med Okayama. 2011;65(2):105-112. 